\title{
Unexplored Potential of Traditional Chinese Medicine in Diabetes Mellitus
}

\author{
Amit Gupta', Tapan Behl'1,* , Arun Kumar ${ }^{1}$, Sukhbir Singh'1, Shaveta Bhardwaj², Amit Goyal ${ }^{3}$ \\ 'Chitkara College of Pharmacy, Chitkara University, Punjab, INDIA. \\ ${ }^{2}$ GHG Khalsa College of Pharmacy, Gurusar Sadhar, Raikot, Punjab, INDIA \\ ${ }^{3}$ Chandigarh College of Pharmacy, Sahibzada Ajit Singh Nagar, Punjab, INDIA.
}

\begin{abstract}
Diabetes is one of the most prevalent disease worldwide and is associated with one of the highest morbidities and mortality rates associated. Like the western and conventional medicines, Traditional Chinese medicine (TCM) has promising results in the ailment of diabetes and its associated complications. But still the use of TCM is refrained for further explorations due to inadequate knowledge of active pharmaceutical ingredient and its isolation. Thus, this review will highlight some of the key concepts and rational behind the therapeutic regimen of TCM aiming towards diabetes treatment. A detailed study of all the articles including research as well as reviews, which were available on the world wide web was performed. The review includes MEDLINE and EMBASE databases using keywords alone or in combinations, such as diabetes, diabetic complications, Chinese therapy, traditional chinese medicine, diabetic neuropathy, diabetic nephropathy, diabetic retinopathy, diabetic complications, $\alpha$-amylase and several others. TCM collectively is an effective and comparatively safe in the treatment of diabetes mellitus and its associated complications. Sig-
\end{abstract}

nificant results are seen in monotherapy and in combination therapy with current conventional drugs. The effects include controlling glycemic levels along with the mitigation of diabetes associated complications. Focus on clinical trials and further investigations might help in concluding TCM as one of the most effective and safe treatment therapies. TCM holds enormous potential by virtue of its mechanism of action by maintaining the homeostasis in the body and thereby exhibiting pharmacological action.

Key words: Diabetic neuropathy, Diabetic nephropathy, Diabetic retinopathy, Anti-inflammatory, Antioxidant, Anti-angiogenic.

\section{Correspondence}

Dr. Tapan Behl

Chitkara College of Pharmacy, Chitkara University, Punjab, INDIA.

Phone no: +91 8527517931

Email: tapanbehl31@gmail.com

DOI: 10.5530/ijpi.2020.1.1

\section{INTRODUCTION}

Diabetes mellitus is one of the most widely spread disorders worldwide. It is a chronic, life-long disorder which affects the endocrine system of the body and affects millions of people every year. In this condition the pancreas does not produce sufficient insulin, or the body cannot effectively utilize insulin that is produced in the human body. Hyperglycaemia or raised blood sugar level, is a common condition of uncontrolled diabetes and its progression can lead to serious damage to many of the body's organ systems, especially the nerves and blood vessels. Some key facts about diabetes from WHO data, Indian Council of Medical Research (ICMR) and International Diabetes Foundation. ${ }^{1}$

- There is an increase in number of people which are having diabetes from 108 million in 1980 to approximately 422 million in 2014 .

- Global prevalence of patients with diabetes in adult population over the age of 18 years has risen from $4.7 \%$ in 1980 to $8.5 \%$ in 2014 .

- In prevalence of diabetes is increasing more rapidly in developing countries within middle and low-income group.

- Blindness, heart attacks, kidney failure, lower limb amputation and stroke are the major complications of diabetes.

- According to a survey in 2016, there were approximately 1.6 million deaths due to diabetes. Also, around 2.2 million deaths were due to hyperglycemia in 2012 .

- It has been estimated that, about half of the total deaths due to high blood glucose, occur before the age of 70 years.
- It is estimated that diabetes was the seventh leading cause of death in 2016.

With the increase in number of population getting affected by diabetes, it has become a matter of great concern to explore novel ways to prevent/ treat this disorder and at the same time reduce the side-effects of other conventional medications used in the treatment of diabetes. Although conventional therapies are effective and satisfactory, but due to the increasing number of side effects associated with these therapies there is an urgent need to develop new drugs derived from natural sources which have less side effects and are as effective as the conventional therapies. ${ }^{2}$ The most commonly occurring side-effects due to some of the conventional therapies for diabetes are described below in Table 1.

Till date, many plants are known to possess antidiabetic activity which includes Cucurbita moschata (Cushaw Seed), Bupleurum chinense (Chinese Thorowax Root), Cinnamomum cassia (Cassia Bark), Coptis chinensis (Coptis Root), Cornus officinalis (Asiatic Cornelian Cherry Fruit), Lonicera japonica Thunb. (Honeysuckle Flower), Scrophularia ningpoensis (Figwort Root), Morus alba (Mulberry Leaf), Ophiopogon japonicaus (Dwarf Lilyturf Tuber), Panax ginseng (Ginseng), Phragmites communis (Reed Rhizome), Dioscorea opposita (Common Yam Rhizome), Eriobotrya japonica (Loquat Leaf), Achyranthes bidentata (Twotooth Achyranthes Root), Alisma orientale (Oriental Waterplantain Rhizome), Anemarrhena asphodeloides (Common Anemarrhena Rhizome), Asparagus cochinchinensis (Cochinchinese AsparagusRoot), Astragaus membranaceus (Milkvetch Root), Atractylodes macrocephala (Largehead Atracty- 
lodes Rhizome), Pinellia ternata (Pinellia Tuber), Polygonum cuspidatum (Giant Knotweed Rhizome), Polygonum multiflorum (Fleeceflower Root), Poria cocos (Indian Bread), Pueraria lobata (Lobed Kudzuvine Root), Rehmannia glutinosa (Chinese Foxglove Root). ${ }^{3}$ The list of plants approved in china is mentioned in Table 2.

\section{DIABETES MELLITUS AND ITS COMPLICATIONS}

Diabetes mellitus is a chronic metabolic disorder which is most commonly characterized by abnormal elevation in blood glucose levels in the body. Uncontrolled diabetes can lead to metabolic disorders which can further lead to acute and chronic complications which may require immediate medical interventions. These complications can lead to morbidity and mortality in diabetic patients.

Based on the case, there are three types of diabetes which are described below:

- IDDM- Type I Insulin dependent diabetes mellitus

- NIDDM-Type II Non-Insulin dependent diabetes mellitus (NIDDM)

- Gestational diabetes which mainly occur during pregnancy only

The other two types of diabetics are:

- Monogenic diabetes- e.g. MODY

Secondary diabetes - e.g. Cushing's disease or acromegaly

Uncontrolled diabetes occurs due to either decreased insulin secretion or impaired insulin signaling pathway or decreased sensitivity of insulin receptors in human body. Basically, there are acute and chronic complications which can affects the human body at various organ system level. ${ }^{4}$ These are described in Figure 1.

Chronic hyperglycaemia may lead to disturbance of homeostasis of the body thereby damaging various cells, of which the most commonly affected cells are the endothelial cells. It has been seen that necrosis of endothelial cells, specially microcapillaries, may lead to damaged vasculature of affected region which can ultimately results in malfunctioning of the

Table 1: Potential side effects of common diabetic drugs.

\begin{tabular}{|c|c|c|}
\hline Sr. No & Category & Side- effects of medication \\
\hline 1 & $\begin{array}{l}\text { Alpha-glucosidase } \\
\text { inhibitor }\end{array}$ & Bloating, gas and diarrhea \\
\hline 2 & Biguanides/Metformin & $\begin{array}{l}\text { Renal complications, upset stomach, } \\
\text { fatigue or dizziness, metallic taste, } \\
\text { Vitamin B12 deficiency, which may cause } \\
\text { anemia and neuropathy (risk in elderly) }\end{array}$ \\
\hline 3 & $\begin{array}{l}\text { Dipeptidyl peptidase } 4 \\
\text { (DPP-IV) inhibitor }\end{array}$ & $\begin{array}{c}\text { Pancreatitis and upper respiratory tract } \\
\text { infection }\end{array}$ \\
\hline 4 & Insulin therapy & $\begin{array}{l}\text { Injection site Lipoatrophy and } \\
\text { lipohypertrophy }\end{array}$ \\
\hline 5 & Meglitinides & Low blood sugar, weight gain \\
\hline 6 & Thiazolidinediones & $\begin{array}{l}\text { Weight gain, increased risk of hepatic } \\
\text { complications, anemia risk, swelling of } \\
\text { limbs }\end{array}$ \\
\hline 7 & $\begin{array}{c}\text { Sodium-glucose } \\
\text { cotransporter (SGLT2) } \\
\text { inhibitor }\end{array}$ & Ketoacidosis (rare), Genital mycosis \\
\hline 8 & Sulfonylureas & $\begin{array}{l}\text { Hypoglycemia, upset stomach, skin rash/ } \\
\text { itching, weight increased }\end{array}$ \\
\hline
\end{tabular}

Table 2: List of Chinese plants with their ingredients approved in China.

\begin{tabular}{|c|c|c|}
\hline $\begin{array}{l}\text { Drug } \\
\text { product }\end{array}$ & Constituents & Family \\
\hline \multirow[t]{3}{*}{ Jin-qi } & Lonicera japonica (Honeysuckle flower) & Caprifoliaceae \\
\hline & Radix astragalus (Milkvetch Root) & Leguminosae \\
\hline & Coptis chinensis (Coptis Root) & Ranunculales \\
\hline \multirow[t]{2}{*}{ Ke-le-nin } & Radix astragalus (Milkvetch Root) & Fabaceae \\
\hline & $\begin{array}{l}\text { Rehmannia glutinosa (Chinese Foxglove } \\
\text { Root) }\end{array}$ & Orobanchaceae \\
\hline \multirow[t]{2}{*}{ Qi-zhi } & Hirudo nipponia (Leech) & Hirudinidae \\
\hline & $\begin{array}{l}\text { Rehmannia glutinosa (Chinese Foxglove } \\
\text { Root) }\end{array}$ & Orobanchaceae \\
\hline \multirow[t]{5}{*}{ Shen-qi } & Panax ginseng (Ginseng) & Araliaceae \\
\hline & $\begin{array}{c}\text { Dioscorea opposita (Common Yam } \\
\text { Rhizome) }\end{array}$ & Dioscoreaceae \\
\hline & Coptis chinensis (Coptis Root) & Ranunculaceae \\
\hline & $\begin{array}{l}\text { Rehmannia glutinosa (Chinese Foxglove } \\
\text { Root) }\end{array}$ & Orobanchaceae \\
\hline & $\begin{array}{c}\text { Cornus officinalis (Asiatic Cornelian } \\
\text { Cherry Fruit) }\end{array}$ & Cornaceae \\
\hline \multirow[t]{4}{*}{ Xiao-ke-an } & Radix astragalus (Milkvetch Root) & Leguminosae \\
\hline & Pueraria lobata (Lobed Kudzuvine Root) & Fabaceae \\
\hline & $\begin{array}{c}\text { Ophiopogon japonicus (Dwarf Lilyturf } \\
\text { Tuber) }\end{array}$ & Asparagaceae \\
\hline & Hirudo nipponia (Leech) & Hirudinidae \\
\hline \multirow[t]{3}{*}{ Yi-jin } & Opuntia dillenii (Cactus) & Cactaceae \\
\hline & $\begin{array}{c}\text { Atractylodes macrocephala (Largehead } \\
\text { Atractylodes) }\end{array}$ & Asteraceae \\
\hline & Poria cocos (Indian Bread) & Polyporaceae \\
\hline \multirow[t]{3}{*}{$\begin{array}{l}\text { Yu-san- } \\
\text { xiao }\end{array}$} & $\begin{array}{l}\text { Rehmannia glutinosa (Chinese Foxglove } \\
\text { Root) }\end{array}$ & Orobanchaceae \\
\hline & Scrophularia ningpoensis (Figwort Root) & Scrophulariaceae \\
\hline & $\begin{array}{c}\text { Anemarrhena asphodeloides (Anemarrhena } \\
\text { Rhizome) }\end{array}$ & Asparagaceae \\
\hline
\end{tabular}

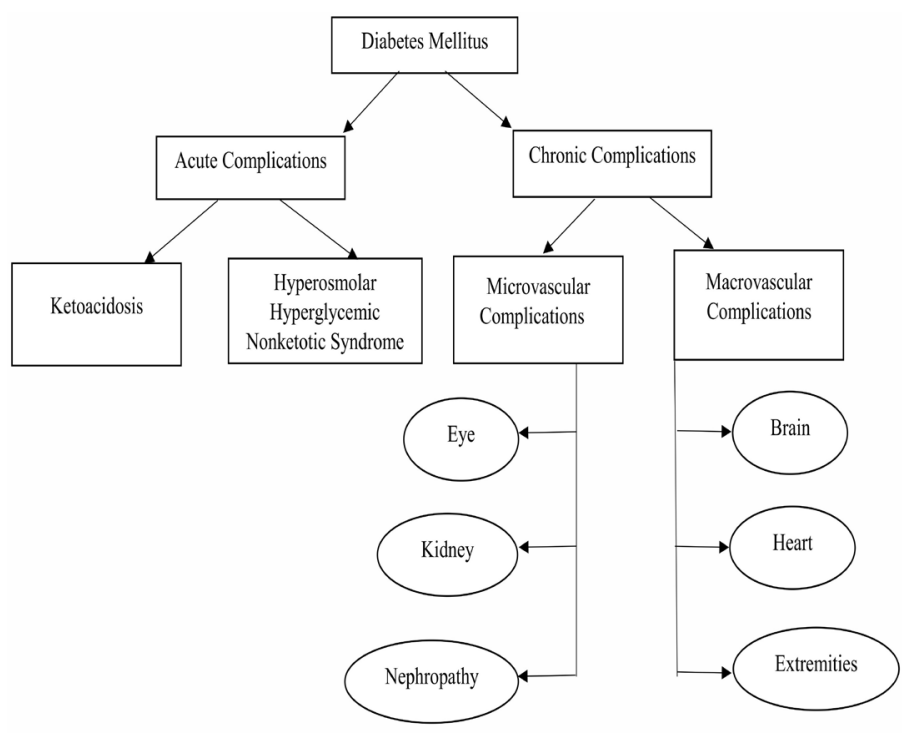

Figure 1: Complications of Diabetes and its effect on organ. 
organ which is being supplied by these blood vessels. These after-effects (chronic/acute) of diabetes are called as diabetic complications. ${ }^{5}$ These complications are named according to the region affected, for instance if the retinal vasculature is affected, it is termed as diabetic retinopathy. Similarly, for neurons, kidney and heart, the complications are named as diabetic neuropathy, diabetic nephropathy and diabetic cardiomyopathy, respectively. ${ }^{6}$ These complications are mainly classified under two categories which are named as macro-vascular and micro-vascular complications, depending upon the size of the blood vessel affected.

In microvascular complications, mainly three organs are affected namely, Eye, Kidney and Nerves (Neuropathy). In eye, high blood glucose along with blood pressure can affect and damage ocular blood vessels, causing cataract, retinopathy and glaucoma. In renal system, it can affect kidneys, due to high blood pressure which can lead to damage of small blood vessels and excess blood glucose overworks the kidney, which results in nephropathy. ${ }^{7}$ It is also known that hyperglycemia damages nerves in the peripheral nervous system (neuropathy) which results in pain and numbness. This can result in gangrene if feet wounds get infected.

Also, macrovascular complications can affect brain, heart and extremities. In brain, it can lead to increased risk of stroke, cerebrovascular disease including transient ischemic attack and cognitive impairment. In cardiovascular system, it can affect heart leading to increased risk of coronary heart disease. In peripheral extremities, diabetes can result in narrowing of blood vessels which can decrease the blood flow in legs. The decrease/reduced blood flow may result in delayed healing and can also contribute to gangrene.

Various studies have been conducted to postulate the exact mechanism underlying diabetic complications. The studies have finally concluded the hyperglycemia mediated effects which can lead to diabetes complications or exacerbation of diabetes. The underlying mechanism for these complications includes increased flux of polyol pathway, overactivation of the protein kinase $\mathrm{C}(\mathrm{PKC})$, increase in production of advanced glycation end products, angiogenesis and inflammation, oxidative stress, among various others. ${ }^{8}$ The combination of these above-mentioned effects may bring out pathological changes which might adversely affect cellular structuring and functioning of human body and can hence play a pivotal role in the pathophysiology of diabetic complications. ${ }^{9}$

\section{THEORIES IN TRADITIONAL CHINESE MEDICINES (TCM)}

This therapy originated thousands of years ago. Since ages, the Chinese therapy including acupuncture/moxibustion, Chinese herbal medicine, dietary therapy and tai chi and qi gong (physical parameters and mental focus) along with mind/body exercises that constitute the backbone of TCM. ${ }^{10}$ TCM originated in China, however, it has spread across the globe and now gaining global recognition.

The therapeutic approach in TCM considers the human body as "holistic" and considers its functioning in a holistic way. TCM is based upon the patterns of disharmony, which include all signs and symptoms presenting patients' emotional and psychological responses. ${ }^{11}$ Although the therapy has been practiced since ages, still the effectiveness of TCM remains questionable. This is mainly due to the fact that Chinese herbal medicine remains poorly researched and very less research has been carried out to explore the active pharmaceutical ingredient (API). Also there are concerns over a number of potentially toxic plants, animal parts and mineral Chinese medicinal which might result in serious side effects. TCM is mainly based on the concept/theories of phenomena that is based in the laws of nature which includes: Yin-Yang Theory and the Five Phases Theory or Five Element Theory.

\section{YIN-YANG THEORY (YINYANG XUE SHUO)}

This is the most fundamental concept of traditional Chinese medicine is Yin and Yang. These are believed to be complementary opposites which are used to describe how things function in relation to each other and to the universe. ${ }^{12}$ In the traditional Yin-Yang symbol, Yin depicts the "dark side" flowing into the Yang called as "light side" and vice versa. The dots within each side symbolize that there is always a bit of Yin within Yang and a bit of Yang within Yin; there are no absolutes. Similarly, in the human physiological functions, signs and symptoms of disease, can be differentiated on the basis of Yin and Yang characteristics following the same principle. ${ }^{13}$

This theory holds a very important place in TCM, as it believes each organ in body has the same element of Yin and Yang within it. For example, some organs, such as the liver, which is predominantly assumed as yang; while the others, such as the kidneys, are considered as yin. Though each organ is assumed to be yin or yang, the homeostasis in these organs is maintained throughout the body, because the sum total of yin and yang will be in balance. ${ }^{14}$

The main mechanism involved the treatment of these diseases is mainly by replenishment of depleted yin or yang. The results of this process bring balance in yin and yang which is ultimaly re-established. The role of medical practitioners using Traditional Chinese medicine mainly focuses on determination of the exact nature of the imbalance, which is then corrected by various approaches, including acupuncture therapy, various herbal remedies, exercising and changes in dietary and lifestyle habits. As soon as the balance is restored in the body, the same moment the person's health is restored. ${ }^{15}$

\section{THEORY OF FIVE PHASES (WUXING)}

The theory of Five Phases, Wu Xing, is a means of classifying phenomena in terms of five basic phases represented by element of wood, fire, earth, metal and water. Those five substances are believed to be the basis elements that comprises everything in universe. ${ }^{16}$

In TCM, it is still believed that there exists a dynamic homeostasis and relationship among these elements and when this balance is interrupted or altered, pathological and physiological changes might occur. The clockwise movement of one element into the next (wood, fire, earth and so forth) whereby one element generates, acts on, or promotes the following element, is referred to as the Sheng cycle. The Ke cycle represents an element acting on or controlling another element in a different order. $^{17}$

As mentioned in the model of Five Phases, each element is associated with an organ. It is based on five principles of wood, fire, earth, metal and water where each element represents different aspects of human body. As per Five Phases theory, wood symbolizes association with hepatic system (liver), fire symbolizes association the cardiac system (heart), earth symbolizes association with spleen-pancreas-stomach, metal with the lungs and water symbolizes association the renal system (kidneys) which is described in Figure 2. In addition, other phenomena, such as seasons, cardinal directions, weather, color and emotions, are associated with each element. Within the TCM model, diagnostic information is gained by finding out patients' favorite season, color and predominant emotion(s).

\section{THEORY OF THE VISCERA AND BOWELS (ZANGFU/ZANGXIANG)}

The term zangxiang is derived from two words i.e Zang and Xiang which in traditional Chinese therapy means viscera referring to internal organs and xiang means phenomena. The word "Zangxiang" thus refers to the 


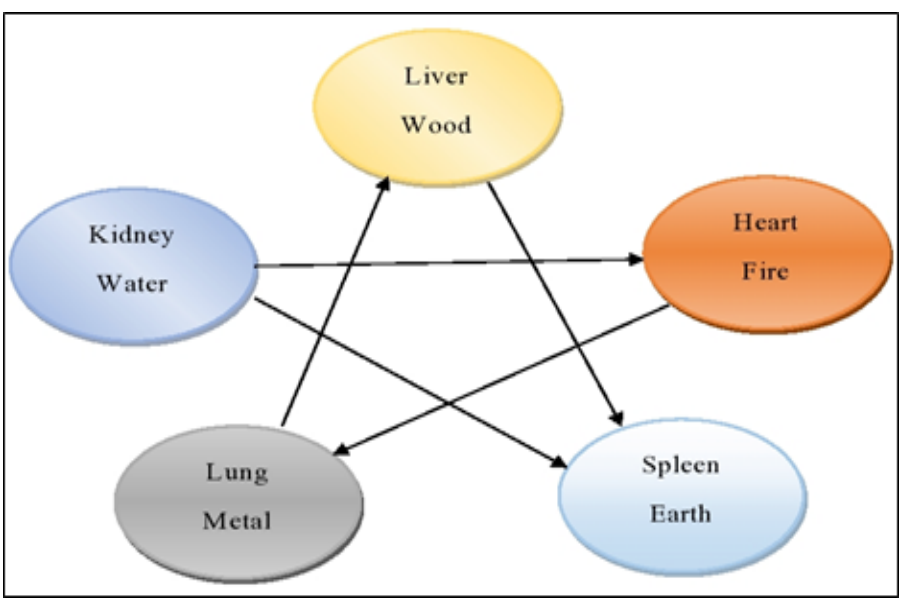

Figure 2: Phenomena restraining five elements in Five Phases Theory.

internal body organs and the external phenomena in pathological and its physiological states.

TCM mentions the human physiology is encoded by sphere of influence which is known as zangfu. This zangfu is described in the western medicine system as 'organs' which interacts in the similar fashion as mentioned in TCM and is responsible for coordination of exterior and internal environment, thereby creating a homeostatic environment. Any change in this function or any disturbance caused by external factors may result in an unbalanced state called as 'disease'.

As mentioned also in Theory of Five Phases (wuxing), the human body is described in five viscera/zang namely: lung, heart, liver, spleen and kidney. According to the theory of Viscera and Bowels, there are six fu (bowels: gallbladder, large intestine, small intestine, stomach, bladder and the Sanjiao) which functions in accordance to the zang constituting the functioning of the human physiological system. ${ }^{18}$ The foundation stone of diagnosis and treatment in TCM is laid by these theories and to maintain the equilibrium in viscera and bowels can help in ailment or treatment of various diseases.

\section{THEORY OF CHANNELS AND COLLATERALS (JINGLUO XUE SHUO)}

This is another theory mentioned in TCM which is based on the same principles of Theory of Five Phases (wuxing) and Theory of the Viscera and Bowels (Zangfu/Zangxiang). In TCM, it has been mentioned that qi and blood circulate throughout the human body in the form of network vessels called as channels and collaterals (jingluo). Like other theories, these channels and collaterals forms a connection between the external and internal environment of human body, creating a loop like system. The imbalance in any, may affect the stability of human body which might create a unbalanced situation 'disease'. These jingluo forms a loop/ circuit and connects each zangfu to each other and also to the extremities of the human body including head and surface of body. It has been postulated that there are twelve main channels called as jing which are associated with zangfu. This interconnections in the form of trajectories led to the foundation and treatment in acupuncture therapy. These channels have a myofascial tract mentioned as "sinew channel," jing jin, a more-detailed "divergent" pathway called as jing bie and a collateral (luo) working in coordination with it.

Additionally, these deep embedded vessels which are known as extraordinary vessels (qi jing ba mai), makes the system complete by acting as a cistern for these twelve main channels. ${ }^{19}$

\section{CHINESE HERBS IN TREATMENT OF DIABETES ASSOCIATED COMPLICATIONS}

Below mentioned are some of the medicinal plants that are being used in Traditional Chinese Medicine since ages, especially for the treatment of diabetes and its associated complications.

\section{YUQUAN WAN}

Yuquan Wan (YQW) also known as "Jade Spring Pills" in Chinese, has been long used to treat diabetes and its associated complications of polydipsia and asthenia in Chinese medicines. In a study carried out in 18 patients with diabetes in 1998, the patients were treated with Yuguan Wan for 1 month, ${ }^{20}$ and it was concluded that $72 \%$ cases showed significant improvement in the fasting blood-glucose and associated diabetic complications of polydipsia. In relation to diabetes associated complications, it has been seen that Yuquan Wan improved the index of kidney injures of early diabetic nephropathy in diabetic patients, which will ultimately delay the development of diabetic nephropathy. In another study carried out by Peng $\mathrm{C}$ et al. it was reported that YQW has promising results in improvement of insulin resistance in patients with T2DM. ${ }^{21}$ In one of the studies carried out by Deng YQ et al. it was elucidated that YQW can significantly reduce the levels of proinflammatory cytokines which are found to be increased in patients with T2DM which can ultimately help in diabetes associated complications of inflammation, tissue destruction and may be helpful in gangrene also. ${ }^{22}$ Also, YQW had a prominent effect on the pharmacokinetics of metformin in animal model of diabetic $^{23}$ which shows that YQW had a better effect than metformin as monotherapy, thus may produce significantly results in improvement of diabetic complications and thereby exhibiting anti-hyperglycemic effect mediated likely by enhancing insulin sensitivity.

\section{TANGMAIKANG JIAONANG}

Tangmaikang Jiaonang (TMK) is one of the herbs which is used to treat T2DM along with its complications. ${ }^{24}$ The anti-diabetic effects are supported by many clinical trials (CT) which confirm its efficacy. ${ }^{25}$ In these CT, TMK was shown to exhibit the effect on insulin resistance, ${ }^{26}$ diabetic peripheral neuropathy, ${ }^{27}$ dyslipidemia ${ }^{28}$ and various blood fluid parameters. ${ }^{29}$ These effects were seen when comparing the baseline values before and after treatment with conventional anti-diabetic therapy. Also, one of the clinical reports in a comparative analysis of these herbs, suggested that TMK exerts better effect in treatment of T2DM patients than Xiaoke Wan. ${ }^{30}$ It was concluded in the study that, TMK could more effectively reduce the blood glucose levels in patients of T2DM when compared to other herbs used in TCM. ${ }^{31}$ Also, along with the monotherapy, the promising effects were seen in combination therapy where TMK in combination with metformin provided better maintenance of glycemic state along with its effects on peripheral neuropathy. ${ }^{32}$ These effects were mediated by increasing the sensitivity of insulin receptors and which may later provide beneficial effects in the treatment of diabetes complications. ${ }^{33}$ TMK did not report any significant adverse effects in patients with T2DM. ${ }^{34}$

\section{XIAOKE WAN}

Xiaoke Wan (XKW) which is known to treat patients with T2DM, comprises of several herb medicines and a well-known anti-diabetic compound, glibenclamide. ${ }^{35}$ The anti-diabetic effect is supported by many clinical reports which proved that XKW had either similar or better effect in treating hyperglycemia in diabetic patients compared with glibenclamide. ${ }^{36} \mathrm{XKW}$ is also known to have pharmacological value due to its effects in the treatment of symptoms associated with diabetic symptoms which includes polydipsia and polyphagia or complications such as 
blood lipid and blood fluid parameters. ${ }^{37}$ It has been reported that XKW not only exert its anti-diabetic activity by stimulation of insulin secretion mainly mediated by glibenclamide (one of components in XKW), but also enhances sensitivity of receptors towards insulin mediated by promoting adiponectin secretion in patients with T2DM. ${ }^{38}$ Although due to the presence of glibenclamide compound, XKW was claimed to be more efficacious and safer than synthetically produced glibenclamide to a certain extent, but herbal medicine components since not validated and purified via proper analytical and extractions methods, the untoward effect of glibenclamide cannot be underestimated. ${ }^{39}$ Special care and attentional should be paid using XKW, since, overuse of this herbal medications due to the presence of glibenclamide can results in well-known side effect of this class of drugs which is severe hypoglycemia which can occur after persistent and overdose. ${ }^{40}$ In a clinical trial which includes 36 patients, use of XKW results it severe hypoglycemic response in T2DM patients, thus dose dependent effect should be well characterized before using XKW. ${ }^{41}$

\section{JINQI JIANGTANG PIAN}

Jinqi Jiangtang Pian (JQJT) is one of the well-known anti-diabetic drugs in TCM but had a mild to moderate antihyperglycemic effect in patient with T2DM. The therapeutic effects may not be significant in patients with T2DM when used as a monotherapy ${ }^{42}$ Therefore, it is used in com- bination with other conventional anti-diabetic drugs which includes glibenclamide. Promising results were seen in these patients who did not show desired effects when using glibenclamide alone. ${ }^{43}$ Thus, it can be postulated the desired effects may be observed when used in combination with Metformin, Acarbose or Glibenclamide and could also show improvement in diabetic dyslipidemia ${ }^{44}$ and preventing the development of diabetic nephropathy. ${ }^{45}$ These effects were confirmed in a clinical trial involving 30 patients. ${ }^{46}$ Also, as a preventive medicine, the effects of JQJT were seen in prediabetes, since prediabetes has become a matter of great concern due to high prevalence of such conditions at an early age. ${ }^{47}$ The anti-diabetic effects are primarily due to improvement of insulin sensitivity and the secondary effects mediated via reduction of serum lipid levels, by regulation of immune system and by triggering the antioxidant pathways. It also helps in improvement of micro-circulation and had effects on beta-cell functioning. ${ }^{48}$ Jinqi Jiangtang Pian did not report any significant adverse effects in patients with T2DM.

\section{JIANGTANGJIA PIAN AND KELENING JIAONANG}

Jiangtangjia Pian (JTJ) is a well-known chinese medicine which is used to treat T2DM patients. In a clinical trial which enrolled 48 T2DM patients, JTJ was found to significantly improved blood glucose level when used in combination with antidiabetic drugs which includes biguanides,

Table 3: Active constituents and mechanism of Chinese drugs.

\begin{tabular}{|c|c|c|}
\hline Chinese herbs & Mechanism of action & Chemical constituents \\
\hline \multirow[t]{5}{*}{ Anisodus tanguticus ${ }^{56}$} & - It is involved in prevention of retinal lipid peroxidation & Anisodamine, anisodine, apoatropine, \\
\hline & $\begin{array}{l}\text { - It acts as a major precursor of downregulating the expression } \\
\text { of plasminogen activator inhibitor-1 }\end{array}$ & $\begin{array}{l}\text { butyryloxytropane, cuscohygrine, hyoscyamine, } \\
\text { scopolamine, trichlorophenyl and tropine. }\end{array}$ \\
\hline & (PAI-1) and tissue factor of fibrinolytic pathway & \\
\hline & - It strongly inhibits the production of cytokine TNF-a & \\
\hline & - It plays a role in downregulation of Vascular peroxidase-1 & \\
\hline \multirow[t]{4}{*}{ Astragalus membranaceus ${ }^{57}$} & $\begin{array}{l}\text { - It helps in decreasing phosphorylation of extracellular signal- } \\
\text { regulated protein kinases (ERK1/2) }\end{array}$ & $\begin{array}{l}\text { Polysaccharides (astragalans I, II and III), saponins } \\
\text { (astragalosides I-VIII and }\end{array}$ \\
\hline & $\begin{array}{l}\text { - It inhibits activation of nuclear factor kappa of activated B cells } \\
\text { and various cytokines }\end{array}$ & $\begin{array}{l}\text { isoastragalosides I and II), flavonoids, isoflavonoids, } \\
\text { sterols, amino acids, volatile oils and trace elements }\end{array}$ \\
\hline & $\begin{array}{l}\text { - It helps in downregulating the expression of enzyme aldose } \\
\text { reductase }\end{array}$ & \\
\hline & $\begin{array}{l}\text { - It helps in reduction of retinal ganglion cell apoptosis in } \\
\text { Glaucoma }\end{array}$ & \\
\hline \multirow[t]{2}{*}{ Ginkgo biloba ${ }^{57}$} & $\begin{array}{l}\text { - It acts as a precursor in downregulating the expression of } \\
\text { Platelet activating factor }\end{array}$ & $\begin{array}{c}\text { Biflavones, flavonol, glycosides, proanthocyanidins, } \\
\text { terpene and trilactones }\end{array}$ \\
\hline & $\begin{array}{l}\text { - Reduce the transcriptional expressions of Hypoxia inducible } \\
\text { factors and vascular and Vascular endothelial growth factor } \\
\text { (VEGF) }\end{array}$ & \\
\hline \multirow[t]{3}{*}{ Puerariae lobate ${ }^{58}$} & - It helps in prevention of peroxynitrite-induced apoptosis & Daidzein, genistein and Puerarin \\
\hline & - It suppresses the activation of NADPH oxidase, & \\
\hline & $\begin{array}{l}\text { - Inhibit tyrosine kinase which acts a precursor by activating } \\
\text { signal transduction cascades }\end{array}$ & \\
\hline \multirow[t]{3}{*}{ Lycium barbarum ${ }^{59}$} & $\begin{array}{l}\text { - It helps in upregulating the expression of anti-apoptotic gene } \\
\qquad \mathrm{Bcl}-2\end{array}$ & $\begin{array}{l}\text { Betaine, beta-sitosterol carotene, cerebroside, } \\
\text { polysaccharides, p-coumaric and zeaxanthin }\end{array}$ \\
\hline & - Inhibit the activation of cytochromes and caspases mediated & \\
\hline & $\begin{array}{l}\text { apoptotic pathway and helps in downregulating the expression } \\
\text { of pro-apoptotic gene Bax }\end{array}$ & \\
\hline \multirow[t]{3}{*}{$\begin{array}{l}\text { Scrophularia } \\
\text { ningpoensis }\end{array}$} & $\begin{array}{l}\text { - It helps in downregulating the expression of Nox-4 and may } \\
\text { exhibit pharmacological action in vascular disorders }\end{array}$ & $\begin{array}{c}\text { Angoroside } \mathrm{C} \text {, acetoside, sibirioside } \mathrm{A} \text {, verbascose } \\
\text { and stachyose }\end{array}$ \\
\hline & - Suppress the expressions of various cytokines & \\
\hline & - It acts by inhibition of COX and may be helpful in arthritis & \\
\hline
\end{tabular}


sulfonylureas and insulin. ${ }^{49}$ The important aspect regarding this study was that the patient's blood glucose was poor controlled by using those anti-diabetic drugs before the use of JTJ. These Herbs of Kelening Jiaonang (KLL) were found to be similar to Jiangtangjia Pian but might have different pharmacodynamic and pharmacokinetic properties. In a clinical trial involving 30 patients, it was reported that KLL can significantly lowered blood glucose level in T2DM patients where it was used in combination with other regular antidiabetic drugs. The results from this trial includes effect compared in before and after treatment with KLL in combination with other conventional drugs. ${ }^{50}$ In another trial involving 33 patients, KLL when given in combination with Glibenclamide as a treatment to T2DM patients, it was reported that it can produces more effective and less toxic effects than monotherapy with glibenclamide. ${ }^{51}$ Another trial involving KLL administration for 1 month was reported to significantly improved blood-glucose-levels in 30 patients of T2DM.52 Adding to this, it was reported that 8 weeks of treatment with KLL can significantly improve blood glucose levels in T2DM patients $(n=21)$ with sulfanylurea failure, which is suggestive that the KLL might improve insulin resistance. ${ }^{53}$ Also, in preclinical studies also, it was confirmed that both JTJ and KLL has tremendous potential to reduce the blood glucose levels in diabetic induced animal models. ${ }^{54}$ All these results from invitro, in-vivo and human trials, strongly suggest that these drugs might exert an insulin-like effects, at the same time being more potent and by less toxic. ${ }^{55}$

The details regarding other Chinese medications its active constituents and its mechanism is mentioned in Table 3.

\section{CONCLUSION}

Just like the "head" and "tail" which corresponds to the two sides of the same coin, TCM also acts in the same manner. The main principal behinds the emergence of TCM is to establish homeostasis in the human body, which is generally in a disturbed state in a diseased condition. On one hand, as proven via clinical trial and animal studies, caution has to be taken while using these drugs owing to the side effects associated with dose related toxicities and its effects on liver. While, on the other hand, it exerts its own beneficial effects too by brining human body to equilibrium by curing or preventing diseased state. Although taking account of the considerations in various researches, there are minimal to no side effects mentioned in numerous scientific reports. Thus, it is very essential to maintain the perfect balance between utilization and disuse of herbal formulations and establish a perfect balance in benefitrisk profile of these drugs. Although in spite of the widespread use of TCM along with its emerging usage in western countries, rigorous scientific evidence of its effectiveness and toxicity profiling is limited. The main difficulty behind the TCM research is due to the complex system of treatment which is based on ideas and concepts which uses altogether a different approach and is very different from that in modern Western medicine. However, the immense potential of this therapy should further be explored and emphasis on toxicogenomic studies can further help in establishment of new regimen which can prove beneficial from the conventional treatment.

\section{ACKNOWLEDGEMENT}

No portion of this manuscript has been submitted for publication elsewhere nor is it under consideration for publication in any other journal, website or textbook. There are no commercial, financial or similar relationships, or corporate appointments of the authors or members of their families to products or companies mentioned in or related to the subject matter of the article being submitted.

\section{CONFLICT OF INTEREST}

The authors declare no conflict of interest.

\section{ABBREVIATIONS}

WHO: World Health Organisation; ICMR: Indian Council of Medical Research; DPP-4: Dipeptidyl peptidase-4; SGLT-2: Sodium-glucose cotransporter-2; IDDM: Insulin dependent diabetes mellitus; NIDDM: Non-Insulin dependent diabetes mellitus; MODY: Monogenic diabetes; PKC: Protein kinase-C; TCM: Traditional Chinese Medicine; API: Active pharmaceutical ingredient; YQW: Yuquan Wan; TMK: Tangmaikang Jiaonang; T2DM: Type 2 diabetes mellitus; XKW: Xiaoke Wan; JQJT: Jinqi Jiangtang Pian; JTJ: Jiangtangjia Pian; KLL: Kelening Jiaonang; PAI-1: Plasminogen activator inhibitor-1; COX: Cyclooxygenase.

\section{REFERENCES}

1. World Health Organisation data. 2019. Available from: http://www.who.int/ news-room/fact-sheets/detail/diabetes

2. Chaudhury A, Duvoor C, Dendi R, Sena V, Kraleti S, Chada A, et al. Clinical review of antidiabetic drugs: Implications for type 2 diabetes mellitus management. Front Endocrinol. 2017;8:6.

3. Jia W, Gao W, Tang L. Antidiabetic herbal drugs officially approved in China. Phytother Res. 2003;17(10):1127-34.

4. Larbie C, Owusu NC, Owusu AC. Phytochemical and Safety Evaluation of Hydroethanolic Leaf Extract of Tecoma stans (L.) Juss. ex Kunth. Evid Based Complement Alternat Med. 2019:7417624

5. Petersmann A, Müller-Wieland D, Müller UA, Landgraf R, Nauck M, Freckmann $\mathrm{G}$, et al. Definition, Classification and Diagnosis of Diabetes Mellitus. Exp Clin Endocrinol Diabetes. 2019;127(S 01):S1-7.

6. Boudina S, Abel ED. Diabetic cardiomyopathy, causes and effects. Rev Endocr Metab Disord. 2010;11(1):31-9.

7. Sharma D, Bhattacharya P, Kalia K, Tiwari V. Diabetic nephropathy: New insights into established therapeutic paradigms and novel molecular targets. Diabetes Res Clin Pract. 2017;128:91-108.

8. Coucha M, Elshaer SL, Eldahshan WS, Mysona BA, El-Remessy AB. Molecular mechanisms of diabetic retinopathy: Potential therapeutic targets. J Ophthalmol. 2015;22(2):135-44.

9. Luis-Rodríguez D, Martínez-Castelao A, Górriz JL, De-Álvaro F, Navarro-González JF. Pathophysiological role and therapeutic implications of inflammation in diabetic nephropathy. World J Diabetes. 2012;3(1):7-18.

10. Atwine F, Hultsjö S, Albin B, Hjelm K. Health-care seeking behavior and the use of traditional medicine among persons with type 2 diabetes in south-western Uganda: A study of focus group interviews. Pan Afr Med J. 2015;20:76.

11. Aung SK, Fay H, Hobbs RF. Traditional Chinese medicine as a basis for treating psychiatric disorders: A Review of theory with illustrative cases. Med Acupunct. 2013;25(6):398-406

12. Kaptchuk TJ. The web that has no weaver: Understanding Chinese medicine. Book Baby; 2nd edition. 2014;45-9.

13. Wang C, Cao B, Liu QQ, Zou ZQ, Liang ZA, Gu L, et al. Oseltamivir compared with the Chinese traditional therapy Maxingshigan-Yinqiaosan in the treatment of H1N1 influenza: A randomized trial. Ann Intern Med. 2011;155(4):217-25.

14. Zhang HJ, Wang ZX. Yin-yang and Zheng: Exported from Chinese medicine. Chin J Integr Med. 2014;20(4):250-5.

15. Gibb C. The Yin and Yang of perioperative aspirin: A clinician's perspective. Int $J$ Cardiol. 2018;258:74-75.

16. Kommonen K. Narratives on Chinese colour culture in business contexts: The yin yang wuxing of Chinese values. Int J Cross Cult Manag. 2011;18(3):366-83.

17. Cao DY. Exploration and analysis of theories of five phases, eight trigrams and four elements. Zhonghua Yi Shi Za Zhi. 2006;36(4):239-42.

18. Liao W, Dang C, Pan W. The brief theory of viscus and its manifestations in traditional Chinese medicine. Integr Med Int. 2017;4(1-2):13-18.

19. Wang J, Wong YK, Liao F. What has traditional Chinese medicine delivered for modern medicine?. Expert Rev Mol Med. 2018;20:e4.

20. Liu SH, Chuang WC, Lam W, Jiang Z, Cheng YC. Safety surveillance of traditional Chinese medicine: Current and future. Drug Saf. 2015;38(2):117-28.

21. Lee AL, Chen BC, Mou CH, Sun MF, Yen HR. Association of traditional Chinese medicine therapy and the risk of vascular complications in patients with type II diabetes mellitus: A nationwide, retrospective, Taiwanese-registry, cohort study. Medicine. 2016;95(3):e2536.

22. Deng YQ, Fan XF, Wu GL. Effect of yuquan pill on proinflammatory cytokines in patients with type 2 diabetes mellitus. Zhongguo Zhong XiYi Jie He Za Zhi. Chin J Integr Med. 2006;26(8):706-9. 
23. Duan HG, WeiYH, Li BX, Zhang DM, Zhang JW, Wu XA. Effects of Yuquan pills on pharmacokinetics of metformin hydrochloride in diabetic rats. Zhongguo Zhong Yao Za Zhi. 2008;5(18):2133-9.

24. Xie $W$, Zhao $Y$, Zhang $Y$. Traditional chinese medicines in treatment of patients with type 2 diabetes mellitus. Evid Based Complement Alternat Med. $2011 ; 2011: 726723$

25. Yue SJ, Liu J, Feng WW, Zhang FL, Chen JX, Xin LT, et al. System pharmacology-based dissection of the synergistic mechanism of Huangqi and Huanglian for diabetes mellitus. Front Pharmacol. 2017;8:694.

26. Wei X, Tao J, Xiao S, Jiang S, Shang E, Zhu Z, et al. Xiexin Tang improves the symptom of type 2 diabetic rats by modulation of the gut microbiota. Sci Rep. 2018;8(1):3685.

27. McGurnaghan SJ, Brierley L, Caparrotta TM, McKeigue PM, Blackbourn LA, Wild SH, et al. The effect of dapagliflozin on glycaemic control and other cardiovascular disease risk factors in type 2 diabetes mellitus: A real-world observational study. Diabetologia. 2019;62(4):621-32.

28. Wang L, LiY, GuoY, Ma R, Fu M, Niu J, et al. Herba Epimedii: An ancient Chinese herbal medicine in the prevention and treatment of osteoporosis. Curr Pharm Des. 2016;22(3):328-49

29. Cai FF, Zhou WJ, Wu R, Su SB. Systems biology approaches in the study of Chinese herbal formulae. Chinese Medicine. 2018;13(1):65

30. Pang B, Ni Q, Lin YQ, Wang YT, Zheng YJ, Zhao XM, et al. Traditional Chinese Patent Medicine for Treating Impaired Glucose Tolerance: A Systematic Review and Meta-Analysis of Randomized Controlled Trials. J Altern Complement Med. 2018;24(7):634-55

31. Yu N, Fang X, Zhao D, Mu Q, Zuo J, Ma Y, et al. Anti-diabetic effects of Jiang Tang Xiao Ke granule via PI3K/Akt signalling pathway in type 2 diabetes KKAY mice. PloS one. 2017;12(1):E0168980.

32. Zan Y, Kuai CX, Qiu ZX, Huang F. Berberine ameliorates diabetic neuropathy: TRPV1 modulation by PKC pathway. Am J Chin Med. 2017;45(08):1709-23.

33. Yue SJ, Liu J, Feng WW, Zhang FL, Chen JX, Xin LT, et al. System pharmacology-based dissection of the synergistic mechanism of Huangqi and Huanglian for diabetes mellitus. Front Pharmacol. 2017;8:694.

34. Ma R, Zhu R, Wang L, Guo Y, Liu C, Liu H, et al. Diabetic osteoporosis: A review of its traditional chinese medicinal use and clinical and preclinical research. Evid Based Complement Alternat Med. 2016;2016:3218313

35. Zhao X, Zhen Z, Guo J, Zhao T, Ye R, Guo Y, et al. Assessment of the reporting quality of placebo-controlled randomized trials on the treatment of Type 2 diabetes with traditional Chinese medicine in mainland China: A PRISMA-compliant systematic review. Medicine. 2016;95(3):e2522.

36. Ji L, Tong $X$, Wang $H$, Tian $H$, Zhou $H$, Zhang $L$, et al. Efficacy and safety of traditional Chinese medicine for diabetes: A double-blind, randomised, controlled trial. PloS one. 2013;8(2):e56703

37. Qiang G, Hengyao Z, Mindian L, Ziyi Z, Yunlin L, Yulei LW, et al. Efficacy and safety of Sancai powder in patients with type 2 diabetes mellitus: A randomized controlled trial. J Tradit Chin Med. 2016;36(5):640-8.

38. Weng J, Ji L, Jia W, Lu J, Zhou Z, Zou D, et al. Standards of care for type 2 diabetes in China. Diabetes Metab Res Rev. 2016;32(5):442-58.

39. Wu JL. Observations into the effects of Xiaoke Wan in treatment of diabetes Shi Yong Zhong Xi Yi Jie He Lin Chuang. 2005;5:18-9.

40. Shan LI. Effects of Xiaoke Wan on serum adiponectin levels in patients with type 2 diabetes mellitus. J Tradit Complement Med. 2010;32(2):112-4

41. Rutebemberwa E, Lubega M, Katureebe SK, Oundo A, Kiweewa F, Mukanga D. Use of traditional medicine for the treatment of diabetes in Eastern Uganda: A qualitative exploration of reasons for choice. BMC Int Health Hum Rights. 2013;13(1):1.
42. Liu Y, Wang A, Wen L, Yang Z, Yang X, Zhang X, et al. A Chinese medicine formula (JinqiJiangtang Tablet): A review on its chemical constituents, quality control, pharmacokinetics studies, pharmacological properties and clinical applications. J Ethnopharmacol. 2019;236:1-8

43. Subramoniam A. Anti-Diabetes Mellitus Plants: Active Principles, Mechanisms of Action and Sustainable Utilization. $1^{\text {st }}$ edition. CRC Press. 2016;199.

44. Min JI. Influence of body differentiation and regulation combining JinqiJiangtang Pian on outcomes of impaired glucose tolerance. Journal of Beijing University of Traditional Chinese Medicine. 2012;9:638-41.

45. Wei CA, Dalian CTC. The Clinical Research into Early Diabetic Nephropathy Treated with Qiming Granule in Combination with Chanchan Shenfeng Decoction. Henan J Tradit Chin Med. 2017(3):47.

46. Zhou F, Chen X. Status of Traditional Chinese Medicine Constitutional Theory in Diabetes Mellitus. J. Tradit Chin Med. 2015(3):15.

47. Gao LH, Liu Q, Liu SN, Chen ZY, Li CN, Lei L, et al. A refined-JinQi-JiangTang tablet ameliorates prediabetes by reducing insulin resistance and improving beta cell function in mice. J Ethnopharmacol. 2014;151(1):675-85.

48. Cao H, Ren M, Guo L, Shang H, Zhang J, Song Y, et al. JinQi-Jiangtang tablet, a Chinese patent medicine, for pre-diabetes: A randomized controlled trial. Trials. 2010;11(1):27.

49. Sun $X$, Guo L, Shang $H$, Ren M, Wang Y, Huo D, et al. The cost-effectiveness analysis of JinQiJiangtang tablets for the treatment on prediabetes: A randomized, double-blind, placebo-controlled, multicenter design. Trials. 2015;16(1):496

50. Shuo C, Jianwei M, Limei X, Tianhui N, Jing D, Wenjun L, et al. Safety and effectiveness of Traditional Chinese Medicinal herbs for diabetic foot: A systematic review and Meta-analysis. J Tradit Chin Med. 2017;37(6):735-45.

51. Ma LX, Wang YY, Li XX, Liu JP. Systematic review on methodology of randomized controlled trials of post-marketing Chinese patent drugs for treatment of type 2 diabetes. Zhong Xi Yi Jie He Xue Bao. 2012;10(3):279-92.

52. Yang HJ, Yao L, Hua JF. Kelening in management of 30 cases of NIDDM. Shanghai Zhong Yi Yao Za Zhi. 1997;9:13.

53. Zhang C. Tangmaikang in treatment of insulin resistance in 26 diabetic patients. Jiang Su Zhong Yi Yao. 2004;25:44.

54. Gong BR, He ZP, Fang YS, Zhu LY. Pharmacodynamics effects of Jiangtangjia Pian. Shi Yan Dong Wu KeXue Yu Guan Li. 2002;19(2):45-6.

55. Yang YP, Huang WZ. Drug efficacy study of Thirst Reliever capsule. Xian Dai Zhong Xi Yi Jie He ZhaZhi. 2007;16:2826-7.

56. Li WL, Zheng HC, Bukuru J, DeKimpe N. Natural medicines used in the traditional Chinese medical system for therapy of diabetes mellitus. J Ethnopharmacol. 2004;92(1):1-21.

57. Li M, Wang W, Xue J, GuY, Lin S. Meta-analysis of the clinical value of Astragalus membranaceus in diabetic nephropathy. J Ethnopharmacol. 2011;133(2):412-9.

58. Chen X, Wang L, Fan S, Song S, Min H, WuY, et al. Puerarin acts on the skeletal muscle to improve insulin sensitivity in diabetic rats involving $\mu$-opioid receptor Eur J Pharmacol. 2018;818:115-23

59. Luo Q, Cai Y, Yan J, Sun M, Corke H. Hypoglycemic and hypolipidemic effects and antioxidant activity of fruit extracts from Lycium barbarum. Life Sci. 2004;76(2):137-49.

60. Hua J, Qi J, Yu BY. Iridoid and phenylpropanoid glycosides from Scrophularia ningpoensis Hemsl. And their $\alpha$-glucosidase inhibitory activities. Fitoterapia. 2014; $93: 67-73$. 